\title{
Charged-particles scattering in the presence of a homogeneous magnetic field
}

\author{
H. S. Brandi and Belita Koiller \\ Departamento de Física, Pontifícia Universidade Católica, Cx.P. 38071, Rio de Janeiro, RJ, Brazil \\ H. G. P. Lins de Barros \\ Centro Brasileiro de Pesquisas Físicas, Rio de Janeiro, RJ, 20000, Brazil \\ L. C. M. Miranda \\ Instituto de Física, Universidade Estadual de Campinas, Campinas, SP, 11300, Brazil \\ (Received 18 July 1977; revised manuscript received 15 November 1977)
}

\begin{abstract}
We study the scattering of charged particles in the presence of a homogeneous magnetic field. Using the Green's function formalism, an appropriate transition amplitude for the scattering process is defined, and application is made on scattering by a Coulomb potential in the high-energy approximation: For this case, the transition amplitude is obtained in a closed form; its behavior with the magnetic field intensity and initial translational energy is qualitatively discussed for transitions from the first Landau level. In the ultrastrongfield limit, the total transition probability presents periodic threshold structures with increasing values of the initial translational energy.
\end{abstract}

\section{INTRODUCTION}

The effect of magnetic fields on the behavior of physical systems is relevant in the study of problems in astrophysics, plasma physics, and solidstate physics, where magnetic fields play an important role in their properties. ${ }^{1-9}$ The structure of matter in the presence of strong magnetic fields has been the subject of several works, ${ }^{1-9}$ and it is a reasonably well-understood problem now: It is known that strong magnetic fields deeply affect the electronic properties of matter. It would be interesting to investigate how transition probabilities are modified by a strong magnetic field, since this could eventually play an important role in physical processes such as population inversion mechanisms, selective excitations of molecules, etc. It is then important to understand how charged particles interact in the presence of a homogeneous magnetic field: To our knowledge there is no theoretical study of this problem.

In the present work we consider the scattering of a charged particle by a structureless potential, and apply the formalism for the specific case of scattering by a Coulomb potential. We also show how the formalism can be extended for scattering by a hydrogen atom, assuming that the eigenfunctions and eigenvalues for the hydrogen atom in the presence of the field are known.

In Sec. II we formulate the problem using the Green's-function approach, and obtain a conveiently defined transition amplitude. Section III is devoted to the study of the results of Sec. II in the case when the scattering potential is of the form
$Z e^{2} / r$, and in Sec. IV we discuss the results obtained.

\section{FORMULATION}

In this section we present the formulation of the problem of a charged particle interacting with a structureless potential in the presence of a homogeneous magnetic field. The eigenstates of a charged particle in a homogeneous magnetic field are known ${ }^{10,11}$ to be separable into a plane wave propagating parallel to the field, and a harmonic oscillator-type solution for the transverse motion, the so-called Landau states. In the usual scattering problems, structureless potentials cannot change the translational energy of the scattered particle. The scattering in the presence of the magnetic field allows a modification of the one-dimensional translation energy caused by a transition between the bound Landau states. It is then possible to define a transition amplitude associated with the process in which a charged particle goes from an initial state $\Phi_{k_{0}, n}$ of wave vector $k_{0}$ and Landau state $n$ into a final state $\Phi_{k, n^{\prime}}$. Our aim is to obtain an expression for this transition amplitude.

Let us consider the Schrödinger equation

$$
\begin{aligned}
H \Psi & =\left[\frac{1}{2 m}\left(\overrightarrow{\mathrm{P}}-\frac{e}{c} \overrightarrow{\mathrm{A}}\right)^{2}+V(\overrightarrow{\mathrm{r}})\right] \Psi \\
& =\left[H_{0}+V(\overrightarrow{\mathrm{r}})\right] \Psi=E \Psi .
\end{aligned}
$$

Taking $\vec{B}=B \hat{z}$ and considering the symmetric Landau gauge, ${ }^{10}$ 


$$
\overrightarrow{\mathrm{A}}=\frac{1}{2} \overrightarrow{\mathrm{B}} \times \overrightarrow{\mathrm{C}},
$$

it is well known that the solution of

$$
H_{0} \Phi=\left(P_{\varepsilon}^{2} / 2 m+H_{\vec{p}}\right) \Phi=\left(E_{0}+\epsilon\right) \Phi
$$

can be separated in the form

$$
\Phi_{k_{0}, n s}(\overrightarrow{\mathrm{r}}) \equiv e^{i k_{0} z} \phi_{n s}(\vec{\rho})
$$

where $E_{0}=\hbar^{2} k_{0}^{2} / 2 m$ and $H_{\vec{p}}$ is the two-dimensional isotropic harmonic-oscillator Hamiltonian $(\vec{\rho}=x \hat{x}$ $+y \hat{y})$ whose eigenfunctions are

$$
\phi_{n s}(\vec{\rho})=(\gamma / \pi)^{1 / 2} e^{i(n-s) \phi} I_{n s}\left(\xi^{2}\right)
$$

with $\xi^{2}=\gamma \rho^{2}$,

$$
\gamma=m \omega_{c} / 2 \hbar \equiv e B / 2 \hbar c \equiv 1 / R_{c}^{2},
$$

and

$$
I_{n s}\left(\xi^{2}\right)=\xi^{|n-s|} e^{-\xi^{2} / 2} Q_{s}^{n-s}\left(\xi^{2}\right)(n ! s !)^{-1 / 2} .
$$

Here $Q_{p}^{\alpha}$ is the associated Laguerre polynomial ${ }^{11}$ conveniently normalized

$$
Q_{s}^{n-s}(x)= \begin{cases}L_{n}^{n-s}(x), & n>s \\ L_{s}^{s-n}(x), & s>n\end{cases}
$$

where $L_{\lambda}^{k}(x)$ is the alternative form of the associated Laguerre polynomial defined in Eq. (d.13) of Ref. 10. The principal quantum number is $n$; $s$ has the geometrical interpretation of the distance of the center of the orbits from the origin. This choice of quantum numbers leads to $0^{10,11}$

$$
\begin{array}{r}
H_{\vec{\rho}} \phi_{n s}(\vec{\rho})=\epsilon_{n} \phi_{n s}(\vec{\rho})=\left(n+\frac{1}{2}\right) \hbar \omega_{c} \phi_{n s}(\vec{\rho}), \\
n=0,1,2, \ldots,
\end{array}
$$

where $\omega_{c}$ is the cyclotron frequency [see (6)] and $\epsilon_{n}$ is the energy of the $n$th Landau level.

Assuming only that the interaction potential $V(\overrightarrow{\mathrm{r}})$ goes to zero for large values of $r$, the assymptotic Green's function can be obtained from

$$
\left(E-H_{\vec{\rho}}-\frac{P_{z}^{2}}{2 m}\right) G\left(\vec{\rho}, \vec{\rho}^{\prime}, z, z^{\prime}\right)=\delta\left(\vec{\rho}-\vec{\rho}^{\prime}\right) \delta\left(z-z^{\prime}\right) .
$$

Let

$$
\begin{aligned}
& G\left(\vec{\rho}, \vec{\rho}^{\prime}, z, z^{\prime}\right) \\
& \quad=\frac{1}{2 \pi} \sum_{n} \int d k \phi_{n s}^{*}\left(\vec{\rho}^{\prime}\right) \phi_{n s}(\vec{\rho}) e^{i k\left(z-z^{\prime}\right)} g_{n}(k) .
\end{aligned}
$$

Substituting (9) in (8) we have

$$
g_{n}(k)=1 /\left[\left(E-\epsilon_{n}\right)-\hbar^{2} k^{2} / 2 m\right]
$$

which leads immediately to

$$
\begin{aligned}
G\left(\vec{\rho}, \vec{\rho}^{\prime}, z, z^{\prime}\right) & \\
& =-\frac{m}{\pi \hbar^{2}} \sum_{n s} \phi_{n s}^{*}\left(\vec{\rho}^{\prime}\right) \phi_{n s}(\vec{\rho}) \int_{-\infty}^{\infty} \frac{d k e^{i k\left(z-z^{\prime}\right)}}{k^{2}-K_{n_{0}, n}^{2}},
\end{aligned}
$$

where

$$
K_{n_{0}, n}^{2}=\left(2 m / \hbar^{2}\right)\left(E-\epsilon_{n}\right)=k_{0}^{2}+\left(2 m / \hbar^{2}\right)\left(\epsilon_{n_{0}}-\epsilon_{n}\right) .
$$

Energy conservation is guaranteed by Eq. (12): the incoming particle with $p_{z}=\hbar k_{0}$ undergoes a transition from the $n_{0}$ to the $n$th Landau level, and $\hbar K_{n_{0}, n}$ is its final momentum parallel to the field. The integration in (11) is equivalent to the one appearing in a one-dimensional problem, ${ }^{13}$ - so that the resulting Green's function is

$$
\begin{aligned}
& G\left(\vec{\rho}, \vec{\rho}^{\prime}, z, z^{\prime}\right) \\
& \quad=-\frac{i m}{\hbar^{2}} \sum_{n s} \phi_{n s}^{*}\left(\vec{\rho}^{\prime}\right) \phi_{n s}(\vec{\rho}) \frac{e^{i K_{n_{0}, n}\left|z-z^{\prime}\right|}}{K_{n_{0}, n}} .
\end{aligned}
$$

Of course the solution of Eq. (1) satisfies

$$
\begin{aligned}
\psi_{k_{0}, n s}(\overrightarrow{\mathrm{r}})= & \Phi_{k_{0}, n s}(\overrightarrow{\mathrm{r}}) \\
& +\int G\left(\overrightarrow{\mathrm{r}}, \overrightarrow{\mathrm{r}}^{\prime}\right) V\left(\overrightarrow{\mathrm{r}}^{\prime}\right) \psi_{k_{0}, n s}\left(\overrightarrow{\mathrm{r}}^{\prime}\right) d \overrightarrow{\mathrm{r}}^{\prime},
\end{aligned}
$$

where $\overrightarrow{\mathrm{r}}=(\vec{\rho}, z)$.

Taking the asymptotic limit, and defining $k$ $\equiv K_{n_{0}, n}$,

$$
\begin{aligned}
\lim _{z \rightarrow \infty} \psi_{k_{0}, n s}(\overrightarrow{\mathrm{r}})= & \Phi_{k_{0}, n s}(\overrightarrow{\mathrm{r}}) \\
& -\frac{i m}{\hbar^{2}} \sum_{n^{\prime} s^{\prime}} \phi_{n^{\prime} s^{\prime}}(\vec{\rho}) e^{i k z} \\
& \quad \times f\left(k_{0}, n s \rightarrow k, n^{\prime} s^{\prime}\right) .
\end{aligned}
$$

The transition amplitude representing an excitation of the $\left(k, n^{\prime} s^{\prime}\right)$ final state from the state $\left(k_{0}, n s\right)$ is defined by

$$
\begin{aligned}
& f\left(k_{0}, n s \rightarrow k, n^{\prime} s^{\prime}\right) \\
& \quad=-\frac{i m}{\hbar^{2} k} \int d \overrightarrow{\mathrm{r}} e^{-i k z} \phi_{n^{\prime} s^{\prime}}^{*}(\vec{\rho}) V(r) \psi_{k_{0}, n s}(\overrightarrow{\mathrm{r}}) .
\end{aligned}
$$

The square of this amplitude is proportional to the probability of finding the particle in a final state $n^{\prime} s^{\prime}$ of momentum parallel to the field $k$, given the initial state of momentum $k_{0}$ and quantum numbers $n s$. This implies that any modification in its momentum in the direction of the field is associated with a transition between Landau levels. Assuming the high-energy (Born) approximation we have

$$
\begin{aligned}
f\left(k_{0}, n s\right. & \left.\rightarrow k, n^{\prime} s^{\prime}\right) \\
& =-\frac{i m}{\hbar^{2} k} \int d \overrightarrow{\mathrm{r}} e^{-i q z} \phi_{n^{\prime} s^{\prime}}^{*^{\prime}}(\vec{\rho}) V(\overrightarrow{\mathrm{r}}) \phi_{n s}(\vec{\rho})
\end{aligned}
$$

and $q=k-k_{0}$ is the transferred momentum in the direction of the field.

The result (17) can be generalized for the scattering of a high-energy charged particle by a hydrogen atom instead of a structureless potential. If the incident particle is an electron, and neglect- 
ing the effect of exchange, we obtain in a similar manner,

$$
\begin{aligned}
& f\left(k_{0}, n s, \beta \rightarrow k, n^{\prime} s^{\prime}, \beta^{\prime}\right) \\
& =-\frac{i m}{\hbar^{2} k} \int d \overrightarrow{\mathrm{r}}_{1} e^{-i q z_{1}} \phi_{n^{\prime} s^{\prime}}^{*}\left(\vec{\rho}_{1}\right) V_{\beta \beta^{\prime}}\left(\overrightarrow{\mathrm{r}}_{1}\right) \phi_{n s}\left(\vec{\rho}_{1}\right),
\end{aligned}
$$

where

$$
\begin{aligned}
V_{\beta \beta^{\prime}}\left(\overrightarrow{\mathrm{r}}_{1}\right)= & -\frac{Z e^{2}}{r_{1}} \delta_{\beta \beta^{\prime}} \\
& +e^{2} \int d \overrightarrow{\mathrm{r}}_{2} \psi_{\beta^{\prime}}^{*}\left(\overrightarrow{\mathrm{r}}_{2}\right) \frac{1}{\left|\overrightarrow{\mathrm{r}}_{1}-\overrightarrow{\mathrm{r}}_{2}\right|} \psi_{\beta}\left(\overrightarrow{\mathrm{r}}_{2}\right) .
\end{aligned}
$$

The wave functions $\psi_{\beta}(\vec{r})$ are the solutions of the Schrödinger equation for a hydrogen atom in a homogeneous magnetic field, and must be obtained in some approximation. ${ }^{2-4,7,8}$

\section{PARTICLE SCATTERING BY A COULOMB POTENTIAL}

For any central field, $V(\vec{r})=V(r)$, integration over the azimuthal angle is immediately done, resulting for the scattering amplitude,

$$
\begin{aligned}
f\left(k_{0}, n s \rightarrow k, n^{\prime} s^{\prime}\right)= & -\frac{i m}{\hbar^{2} k} \delta_{n-s, n^{\prime}-s^{\prime}} \\
\times & \times \int_{0}^{\infty} d x \int_{-\infty}^{\infty} d z e^{-i \alpha z} I_{n^{\prime} s^{\prime}}(x) I_{n s}(x) \\
& \quad \times V\left(\left(x / \gamma+z^{2}\right)^{1 / 2}\right),
\end{aligned}
$$

where $x=\gamma \rho^{2}$, with $\gamma$ given by $(6)$, and $\left(\rho^{2}+z^{2}\right)^{1 / 2}$ $=r$.

Considering the specific case of the Coulomb potential and the fact that the potential is even in the $z$ coordinate, we have

$$
f\left(k_{0}, n s \rightarrow k, n^{\prime} s^{\prime}\right)=-\frac{2 i m}{\hbar^{2} k} Z e^{2} \delta_{n-s, n^{\prime}-s^{\prime}} \int_{0}^{\infty} d x \int_{0}^{\infty} d z \cos q z \int_{0}^{\infty} d y e^{-y z} I_{n^{\prime} s^{\prime}}(x) J_{0}\left(y(x / \gamma)^{1 / 2}\right) I_{n s}(x),
$$

where we have used the fact that for $z>0$

$$
\frac{1}{\left(\rho^{2}+z^{2}\right)^{1 / 2}}=\int_{0}^{\infty} d y e^{-y z} J_{0}(y \rho) .
$$

After performing the integration over $z$, Eq. (21) becomes

$$
\begin{aligned}
f\left(k_{0}, n s \rightarrow\right. & \left.k, n^{\prime} s^{\prime}\right) \\
= & -\frac{2 Z e^{2} m}{\hbar^{2} k} \delta_{n-s, n^{\prime}-s^{\prime}} \int_{0}^{\infty} \frac{y d y}{y^{2}+q^{2}} \\
& \times \int_{0}^{\infty} I_{n^{\prime} s^{\prime}}(x) I_{n s}(x) J_{0}\left(y(x / \gamma)^{1 / 2}\right) d x .
\end{aligned}
$$

Using the relation ${ }^{11}$

$$
\begin{aligned}
\int_{0}^{\infty} d x I_{\alpha \beta}(x) I_{\gamma \delta}(x) J_{(\alpha-\beta)-(\gamma-\delta)} & (2 \sqrt{A x}) \\
= & I_{\alpha \gamma}(A) I_{\beta} \cdot(A),
\end{aligned}
$$

integration over $x$ is readily done, yielding

$$
\begin{aligned}
& f\left(k_{0}, n s \rightarrow k, n^{\prime} s\right) \\
& \quad=-\frac{i m Z e^{2}}{\hbar^{2} k} \delta_{n-s, n^{\prime}-s^{\prime}} \int_{0}^{\infty} \frac{d x}{x+\lambda} I_{n^{\prime} n}(x) I_{s^{\prime} s}(x),
\end{aligned}
$$

where $\lambda=q^{2} / 4 \gamma$.

From the definition of Laguerre polynomials, we get

$$
\begin{aligned}
I_{n^{\prime} n}(x) I_{s^{\prime} s}(x)=e^{-x} \sum_{m=0}^{\bar{M}_{1}} \sum_{p=0}^{\bar{M}_{2}}(-1)^{m+p_{1}} \\
\quad \times c\left(n^{\prime} n s^{\prime} s m p\right) x^{m+p+|n-s|},
\end{aligned}
$$

where the coefficients $c\left(n^{\prime} n s^{\prime} s m p\right)$ are well defined, ${ }^{10}$ $\bar{M}_{1}$ is the largest between $n$ and $n^{\prime}$, and $\bar{M}_{2}$ the largest between $s$ and $s^{\prime}$. Using this expansion, Eq. (25) can be integrated (Ref. 12, Eq. 3.3535, p. 310), yielding

$$
\begin{aligned}
f\left(k_{0}, n s \rightarrow k, n^{\prime} s^{\prime}\right)=i \frac{m Z e^{2}}{\hbar^{2} k} \delta_{n-s, n^{\prime}-s^{\prime}}\left(I_{n^{\prime} n}(-\lambda) I_{s^{\prime} s}(-\lambda) \operatorname{Ei}(-\lambda)\right. & \\
& +(-1)^{|n-s|+1} \sum_{m=0}^{\bar{M}_{1}} \sum_{p=0}^{\bar{M}_{2}} \sum_{l=1}^{m+p+\bar{M}_{1}+\bar{M}_{2}}(-1)^{m+p-1} c\left(n^{\prime} n s^{\prime} \operatorname{smp}\right)(l-1) ! \lambda^{m+p+|n-s|-\imath) .}
\end{aligned}
$$

$\operatorname{Ei}(-\lambda)=-E_{1}(\lambda)$ is the exponential integral function. ${ }^{12}$

For sake of simplicity, we consider for discussion the case when the initial state has $n=0=s$. In this case (27) reduces to

$$
\begin{aligned}
f\left(k_{0}, 00 \rightarrow k, n^{\prime} s^{\prime}\right) & =i \frac{m Z e^{2}}{\hbar^{2} k}\left[I_{n^{\prime} 0}(-\lambda)\right]^{2} \operatorname{Ei}(-\lambda) \\
& =-i \frac{m Z e^{2}}{\hbar^{2} k}\left[Q_{n^{\prime}}^{n^{\prime}}(-\lambda)\right]^{2} e^{\lambda} E_{1}(\lambda) .
\end{aligned}
$$


Since $Q_{n^{\prime}}^{n^{\prime}}(x)=(-1)^{n^{\prime}} n^{\prime} !$,

$f\left(k_{0}, 00 \rightarrow k, n^{\prime} s^{\prime}\right)=-i \frac{m Z e^{2}}{h^{2}} \frac{\left(n^{\prime} !\right)^{2} e^{\lambda} E_{1}(\lambda)}{k}$.

We present a simple qualitative discussion about the dependence of the transition amplitude on the magnetic field intensity and on the incident translational energy $E_{0}=\hbar^{2} k_{0}^{2} / 2 m$. Energy conservation implies that transitions occur only if $\Delta n \hbar \omega_{c} \leqslant E_{0}$ $\left(\Delta n=n^{\prime}-n\right)$. Since we assume $n=0$, our discussion is restricted to excitation of Landau levels, $\Delta n>0$, so that the relevant parameters of the problem may assume the following corresponding ranges of values:

$$
0 \leqslant \lambda \leqslant \Delta n-k_{0} \geqslant k \geqslant 0-\infty \geqslant E_{0} / \hbar \omega_{c} \geqslant \Delta n .
$$

The absolute value of the transition amplitude given by (29) for a fixed $\Delta n$ decreases monotonically with the parameter $E_{0} / \hbar \omega_{c}$, and because of the factor $k^{-1}$, it diverges for $E_{0} / \hbar \omega_{c}=\Delta n$, which is the threshold condition. Of course for $E_{0} / \hbar \omega_{c}<\Delta n$ it vanishes. Making use of the inequality ${ }^{14}$

$$
1 /(x+1)<e^{x} E_{1}(x) \leqslant 1 / x \text { for } x>0,
$$

it can be shown that the absolute value of the transition amplitude increases with $\Delta n$. The behavior is qualitatively shown in Fig. 1 . The total transition probability from the initial state $(n, s)=(0,0)$ : $P_{0}=\sum_{n^{\prime}} \mid f\left(k_{0}, 00 \rightarrow k,\left.n^{\prime} s^{\prime}\right|^{2}\right.$ as a function of $E_{0} / \hbar \omega_{c}$ should therefore present periodic threshold structure, separated by unity, corresponding to a process where the initial translational energy $E_{0}$ is totally transferred into a Landau-level excitation. The total transition probability $P_{0}$ as a function of $E_{0} / \hbar \omega_{c}$ has an overall increasing oscillatory behavior, as shown in Fig. 1. It should be noted that effects such as the de Haas-van Alphen and Schubnikow-van Alphen present a similar behavior for the magnetic susceptibility and the electric resistivity, respectively, as a function of the inverse of the magnetic field. ${ }^{15}$

For transitions from initial states others than the one discussed above, the analytic expression of the transition amplitude is given by Eq. (27). The periodic structures in the total transition probability are still present, because of the factor $k^{-1}$ in the first term of that equation. Although we have not carried out a detailed analysis of the function (27), we believe that the general behavior of the transition amplitude and total transition probability for excitation of Landau levels also present the aspect depicted in Fig. 1. The overall increasing behavior of the total transition probability with the incident energy is due to the larger number of accessible final states; notice that each transition amplitude between two particular states is a decreasing function of $E_{0} / \hbar \omega_{c}$.
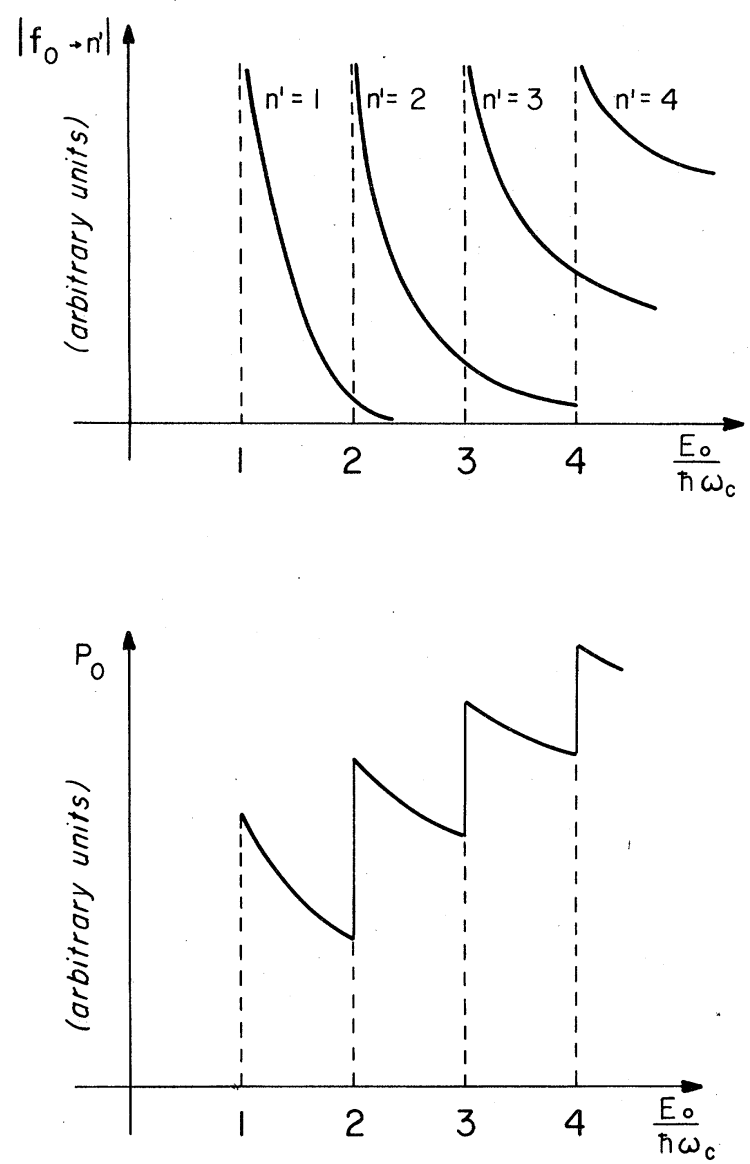

FIG. 1. Qualitative dependence of the partial transition amplitude $\left|f_{0} \rightarrow n^{\prime}\right|$ and of the total transition probability from state $(n, s)=(0,0), P_{0}$, as a function of the initial translational energy relative to the Landau-level separation.

\section{CONCLUSIONS}

The scattering of charged particles in the presence of a homogeneous magnetic field has been studied, and an application was made for the case of scattering by a Coulomb potential in the highenergy approximation. The presence of the magnetic field allows for inelastic processes (transition between Landau levels) even when the scattering is associated with a structureless potential. Due to the separable form of the solution (4) this problem is formally equivalent to the one-dimensional scattering of a charged particle (characterized by it momentum $\hbar k$ ) by a system associated with the Landau levels.

For collisions of charged particles in the presence of a homogeneous magnetic field, a typical value of the translational energy (within the Born approximation) is $E_{0}=100 \mathrm{eV}$. This corresponds to the separation between Landau levels in a mag- 
netic field of the order of $10^{10} \mathrm{G}$. The threshold structure we have previously discussed can only be observed for fields of that order or larger, which are presently known to exist in the surface of neutron stars and pulsars.

A field of $10^{10} \mathrm{G}$ is orders of magnitude larger than any experimentally available magnetic field. Strong magnetic fields in the laboratory are of the order of $10^{5} \mathrm{G}$, so that the parameter $E_{0} / \hbar \omega_{c}$ is about $10^{5}$ for $E_{0}=100 \mathrm{eV}$. Separation between structures of the order of $10^{-5}$ of the value of the parameter can not be experimentally observed, and the only observable effect in the laboratory should be a slow increase of $P_{0}$ with $E_{0} / \hbar \omega_{c}$. Therefore the total transition probability increases with $E_{0}$ and decreases with $B$; it is only in the ultrastrongfield limit $\left(B \gtrless 10^{10} \mathrm{G}\right)$ that the increasing oscillatory behavior can be observed.

The scattering of a charged particle by a hydrogen atom in the presence of a magnetic field is more complicated, since the dependence of the atomic levels as a function of the magnetic field is not known in an analytic form, as the Landau levels, and the general behavior depends on the level considered $^{2-4,7,8}$; no general behavior can be anticipated without specific calculations.
${ }^{1}$ R. Cohen, J. Lodenquai, and M. Ruderman, Phys. Rev. Lett. 25, 467 (1970).

${ }^{2}$ E. R. Smith, R. J. W. Henry, G. L. Surmeliau, R. F. O'Connell, and A. K. R. Rajagopal, Phys. Rev. D $\underline{6}$, 3700 (1972).

${ }^{3}$ A. R. P. Rau, R. O. Mueller, and L. Spruch, Phys. Rev. A 11, 1865 (1975); A. R. P. Rau and L. Spruch, Astrophys. J. 207, 671 (1976).

${ }^{4}$ H. S. Brandi, Phys. Rev. A 11, 1835 (1975); R. R. dos Santos and H. S. Brandi, ibid., 1970 (1976); H. S. Brandi, R. R. Santos and L. C. M. Miranda, Lett. Nuovo Cimento 16, 187 (1976).

${ }^{5}$ B. B. Kadomtsev, Zh. Eksp. Teor. Fiz. 58, 1765 (1970) [ Sov. Phys. JETP 31, 945 (1970)]; B. B. Kadomtsev and V. S. Kudryavtsev, Pis' ma Zh. Eksp. Teor. Fiz. 13, 61 (1970) [JETP Lett. 13, 42 (1971)].

${ }^{6}$ C. P. de Melo, R. Ferriera, H.S. Brandi, and L. C. M. Miranda, Phys. Rev. Lett. 37, 676 (1976).

${ }^{7}$ Y. Yafet, R. W. Keyes, and E. N. Adams, J. Phys.
Chem. Solids 1 , 137 (1956).

${ }^{8}$ H. S. Praddaude, Phys. Rev. A 6, 1321 (1972).

${ }^{9} \mathrm{M}$. Ruderman, in IAU Symposium No. 53 , Physics of Dense Matter, edited by C. Hansen (Reidel, Holland, 1974), p. 117.

${ }^{10} \mathrm{~L}$. Landau and E. Lifchitz, Mécanique Quantique (Editions Mir, Moscow, 1966).

${ }^{11}$ A. A. Sokolov and I. M. Ternov, Syncroton Radiation (Pergamon, New York, 1968); V. Canuto and D. C. Kelly, Astrophys. Space Sci. 17, 277 (1972).

${ }^{12}$ I. S. Gradshteyn and I. M. Ryzlhik, Tables of Integrals, Series and Products (Academic, New York, 1965).

${ }^{13} \mathrm{R}$. J. Glauber, Lectures in Theoretical Physics (Interscience, New York, 1959), Vol. I, p. 333.

${ }^{14}$ M. Abramowitz and I. A. Segun, Handbook of Mathematical Functions (Dover, New York, 1968).

${ }^{15} \mathrm{C}$. Kittel, Quantum Theory of Solids (Wiley, New York, 1963). 